Supporting Information

\title{
A General Method for Assembling Single Colloidal Particle Lines
}

Jiaxing Huang, Andrea R. Tao, Stephen Connor, Rongrui He and Peidong Yang*

\author{
Department of Chemistry, University of California, Berkeley \\ Materials Science Division, Lawrence Berkeley National Laboratory \\ Berkeley, CA 94720 \\ *Email: p_yang@berkeley.edu
}

\section{Methods}

Preparation of nanoparticle monolayers. Gold $(50-100 \mathrm{~nm})^{1}$, silver $(\sim 50 \mathrm{~nm})^{2}$ and platinum $(\sim 7 \mathrm{~nm})^{3}$ nanoparticles were prepared based on a modified polyol process as described elsewhere, using poly(vinyl pyrrolidone) (PVP, Mw 55,000) as the surface protecting agent and ethylene glycol or 1, 5-pentanediol as both the solvent and reducing agent. Excess PVP and unreacted salts were removed by centrifugation. Silica spheres $(0.16-2.3 \mu \mathrm{m}$, Bangs Laboratories Inc.) were first coated with a positively charged polyelectrolyte, poly(diallyl dimethylammonium chloride) layer ${ }^{4}$ to ensure good adhesion of PVP, which renders good dispersibility in chloroform. The particles were then redispersed in chloroform by solvent exchange. Then, a small volume of the chloroform dispersion, typically 1 to $2 \mathrm{ml}$, was spread onto a water surface in a rectangularshaped Teflon ${ }^{\circledR}$ trough with an area of $250 \mathrm{~cm}^{2}$ (NIMA 611D with a PS4 surface pressure sensor).

Single particle line deposition. Silicon and glass wafers (typically $1 \times 1$ or $1 \times 2 \mathrm{~cm}^{2}$ ) were either used as received, or cleaned with acetone by sonication. Some silicon substrates were treated in a high temperature oven $\left(1000^{\circ} \mathrm{C}\right)$ to grow a dense thermal oxide layer. A substrate was then mounted on a dip-coater and dipped into the water subphase. A raising speed of 1 to $30 \mathrm{~mm} / \mathrm{min}$ was used for the coating experiments.

Growth of 1D arrays of silicon nanowires. Silicon nanowires were grown by chemical vapor deposition using $\mathrm{SiCl}_{4}$ as the precursor and $\mathrm{H}_{2}$ (10\% in argon) as the carrier gas ${ }^{5}$. Silicon (111) wafers with pre-deposited gold single particle lines were placed in a tube furnace and heated up to $850{ }^{\circ} \mathrm{C}$ for 15 minutes for the nanowire growth. 


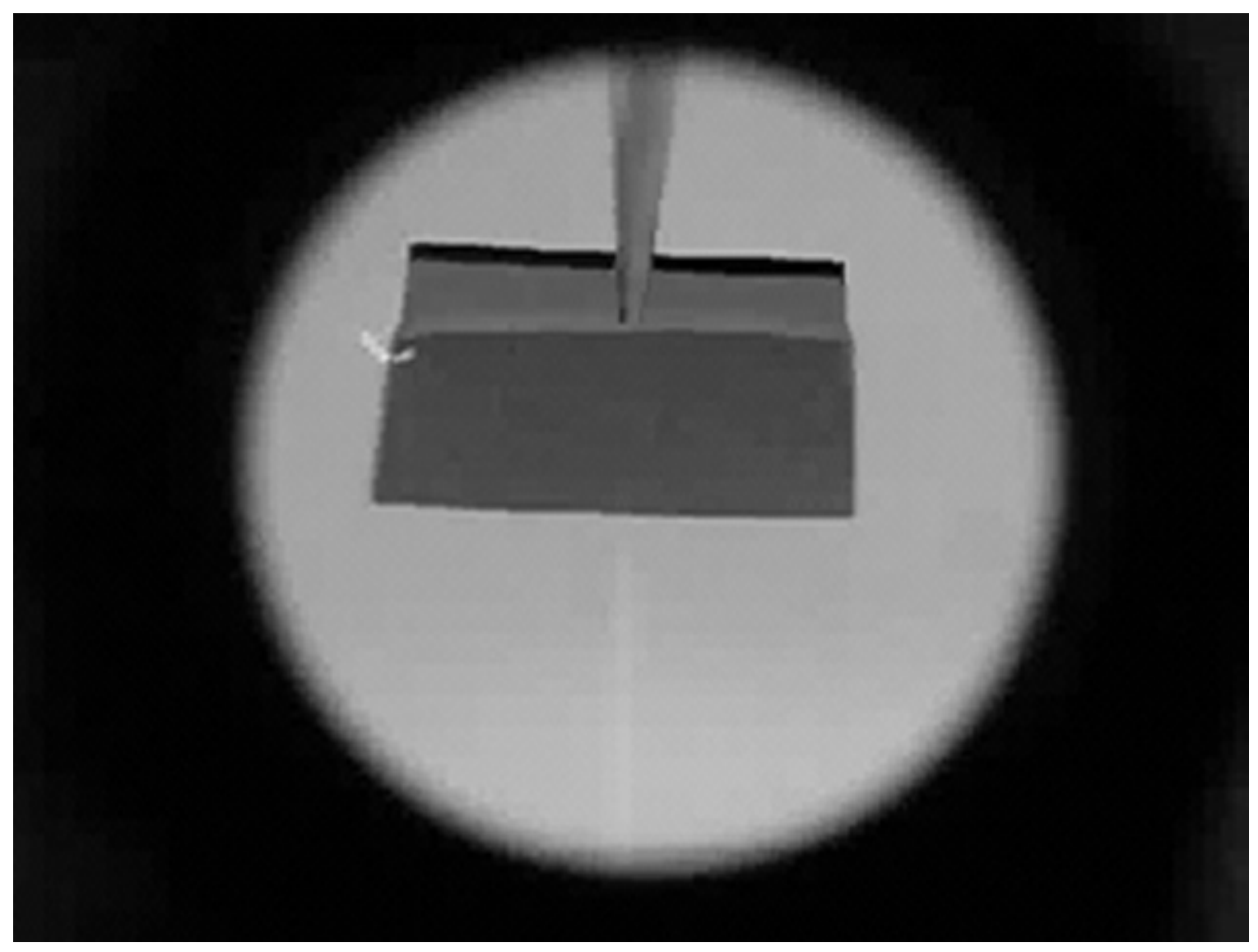

Video S1 Stick-slip motion of the water-substrate contact line as the substrate is raised through a monolayer of $\mathrm{SiO} 2$ spheres on a water surface. The substrate is a $1 \times 1 \mathrm{~cm}^{2} \mathrm{Si}$ wafer. The playback rate of the movie is 12 times of the recording speed. 


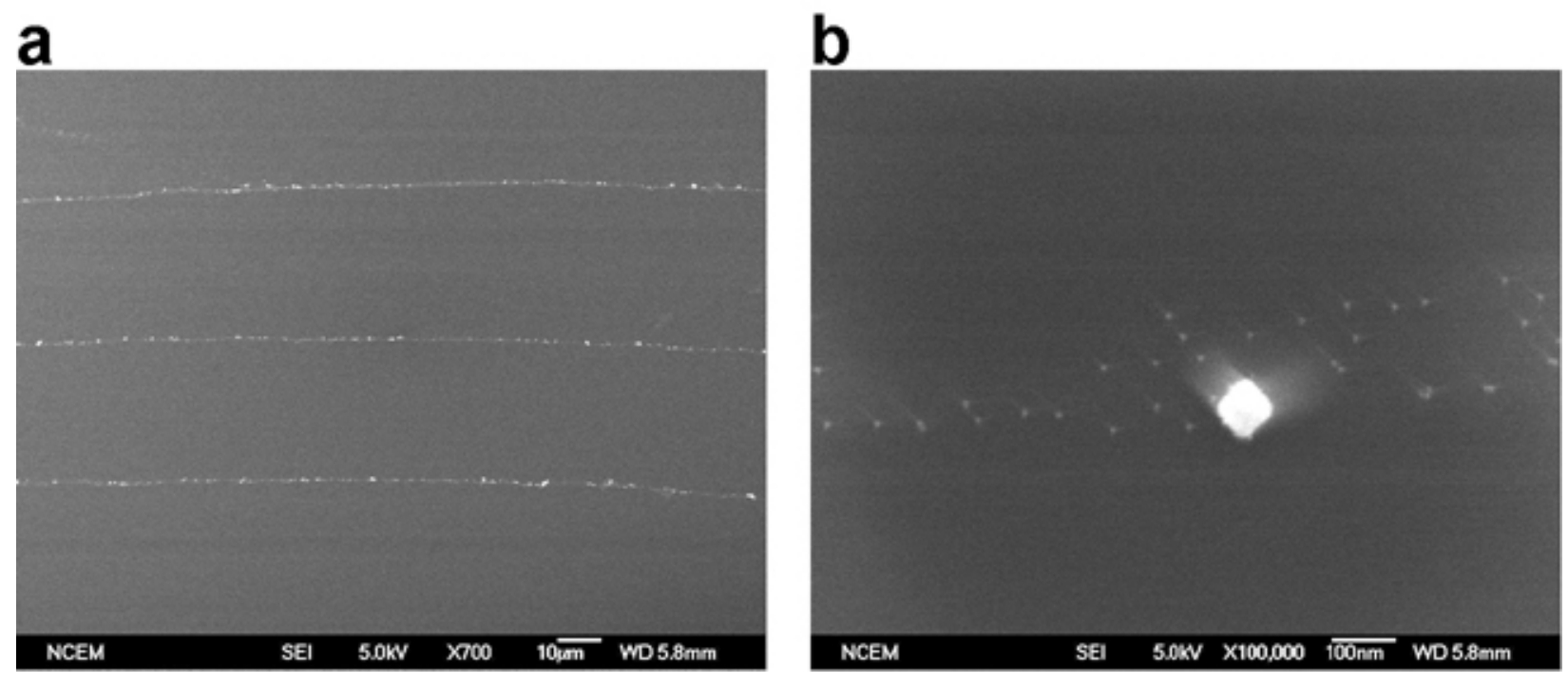

Figure S1 SEM images showing (a) an overview and (b) a close up of 1D arrays of particles with different sizes: $\sim 80 \mathrm{~nm}$ Au and $\sim 7 \mathrm{~nm}$ Pt nanoparticles. 


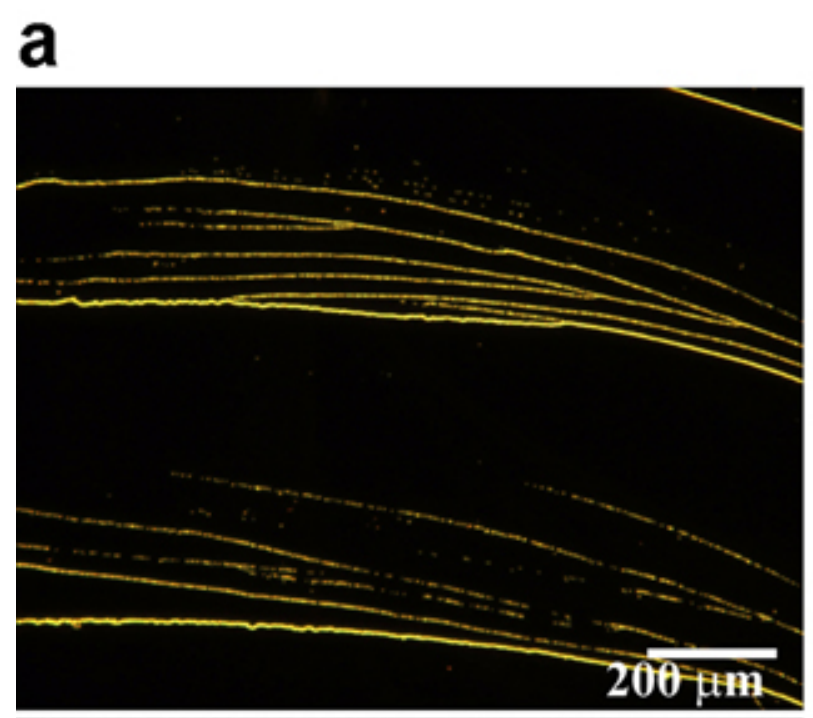

\section{b}
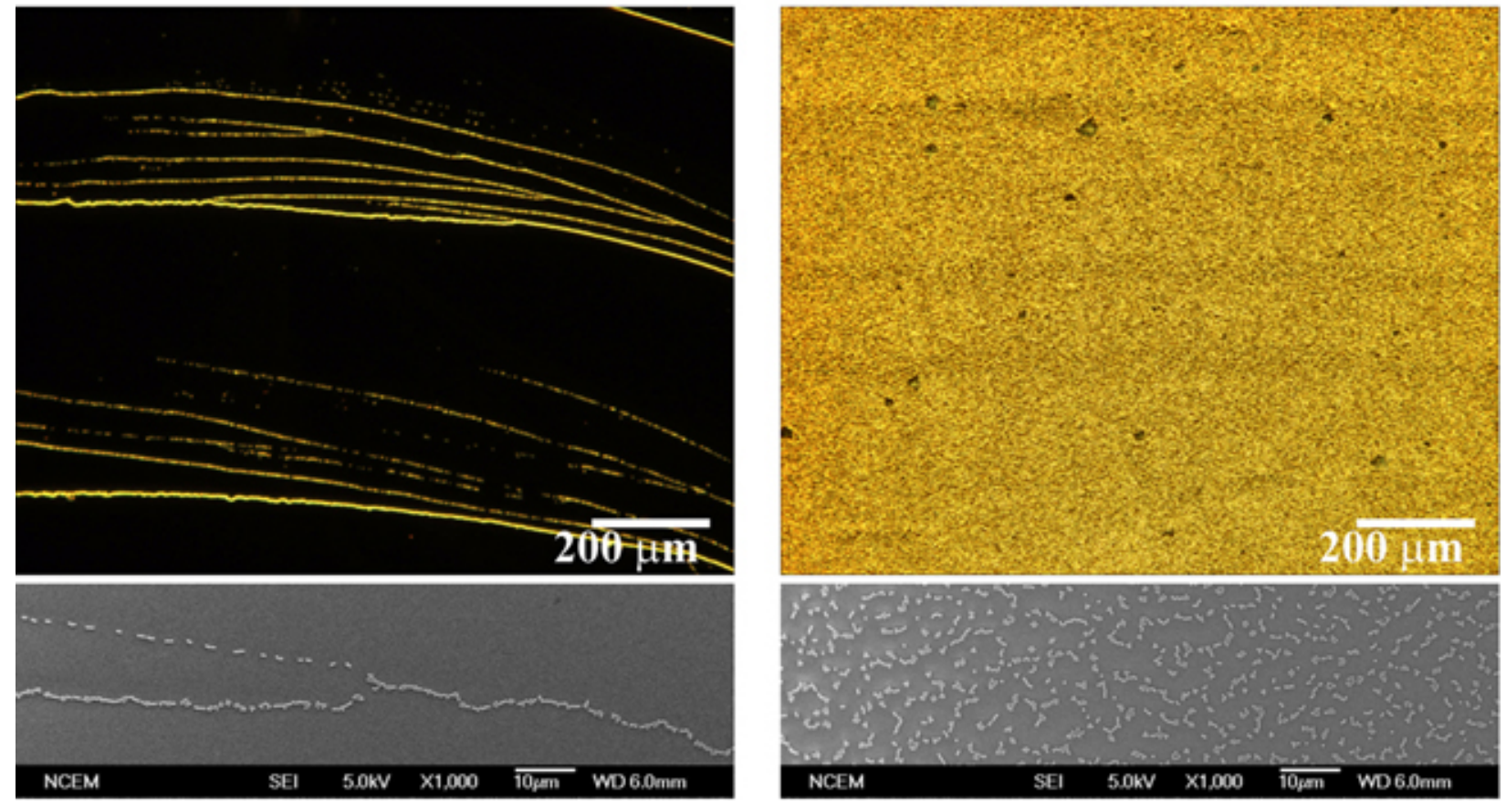

Figure S2 Optical (top) and scanning electron microscopy (bottom) images showing (a) branched lines and (b) continuous film collected at point $\mathrm{E}\left(60 \mathrm{~cm}^{2}\right)$ and $\mathrm{F}\left(50 \mathrm{~cm}^{2}\right)$ on the isotherm in Figure 2a, respectively. 


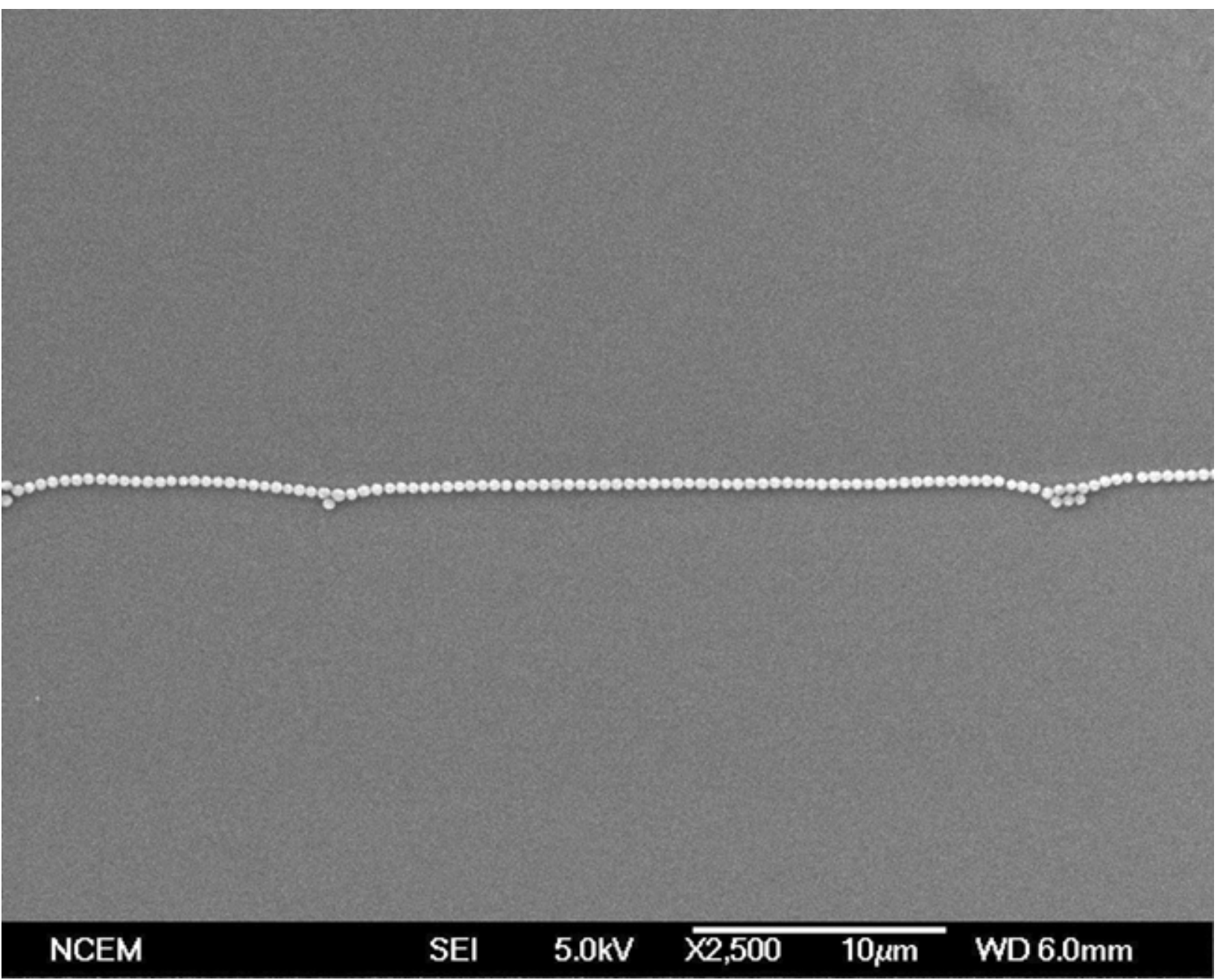

Figure S3 Longer colloidal particle oligomers, even "polymers" such as the one shown in the SEM image that consists of more than 130 colloidal particles, can be obtained by increasing the "sticking" time of the contact line.

1. Kim, F.; Connor, S.; Song, H.; Kuykendall, T.; Yang, P. Angew. Chem. Int. Ed. 2004, 43, (28), 3673-3677.

2. Sun, Y.; Xia, Y. Science 2002, 298, (5601), 2176-2179.

3. Song, H.; Kim, F.; Connor, S.; Somorjai, G. A.; Yang, P. D. J. Phys. Chem. B 2005, 109, (1), 188-193.

4. $\quad$ Decher, G. Science 1997, 277, (5330), 1232-1237.

5. Hochbaum, A. I.; Fan, R.; He, R.; Yang, P. Nano Lett. 2005, 5, (3), 457-460. 